\title{
Utilization of consumer electronics for an economically affordable motorized wheelchair
}

\section{Benedikt Prusas a , Karim Mansoor, L. Engelhardt, Saku Pirtilä, Bas Walgers, Louisa Lukoschek, Ismael Lengua ${ }^{\mathrm{b}}$, María Moncho-Santonja ${ }^{\mathrm{b}}$ and Guillermo Peris- Fajarnés $^{\text {b* }}$}

${ }^{\mathrm{a}}$ Hochschule Düsseldorf, Germany, Benedikt.Prusas@ @study.hs-duesseldorf.de, ${ }^{\mathrm{b}} \mathrm{CITG}$ - Universitat Politècnica de Valéncia, Valencia, Spain, *e-mail: gperis@upv.es

\begin{abstract}
A design (sketch) of a prototype electric scooter adaptable to a wheelchair is proposed. A mechanism that allows adapting an electric power unit to a conventional wheelchair is presented. The aim of this design is to create an economic solution to motorize a wheelchair and support the independent mobility of wheelchair users. This is especially relevant as the number of wheelchair users is increasing. The device consists of a self-balancing scooter, serving as the power unit, which is replacing the main wheels of the wheelchair and a metal link, connecting it to the wheelchair. The steering is controlled with two sticks, which directly exerts pressure on the sensor pad and steering unit of the self-balancing scooter. By using a self-balancing scooter and hardware store materials the costs can be kept low and accessible to many people.
\end{abstract}

Keywords: motorized wheelchair, electric powered, self-balancing scooter, mobility

\section{Introduction}

The world population is growing and the demographic transition is in place, increasing the number of elderly people (Division., 2017). Thus, the need for wheelchairs is increasing by approximately 3500 units per day (Foundation, s.f.). And given that the quality of life is directly correlated to the mobility of a person (Hudakiva \& Hornakova, 2011) making mobility accessible is an important topic. The world report on ageing and health states: 
"When assistive devices are available, affordable and appropriate to older people's needs and their environments, their mobility, independence and participation can be greatly enhanced." (Organisation, 2015). Progress and efforts have been made in this regard. Such as of the wheelchair foundation which distributed over a million wheelchairs (Foundation, s.f.) or innovative devices such as the all-terrain wheelchair, developed by Amos Winter (Winter, 2014). Also, attempts have been made to make power assisted mobility solutions more accessible as well. For example, students of the Bingham Young University have founded the Open Wheelchair Foundation and published instructions to build an electric wheelchair (Hollingshead, 2015).This paper is another contribution to explore options to make power assisted mobility more accessible. Especially for those cases where the physical condition of the person is not sufficient to operate a manual wheelchair and is covering the question of whether there is a way to make motorized wheelchair solutions more affordable. This work has been created in the scope of a university project restricted by a four months-long time frame and a limited budget. Based on those limitations the decision was made, to utilize the benefits from mass production of already existing consumer electronics. Chosen was a self-balancing scooter. The requirements for the design include that it can be used in the daily routine and among other things to being able to turn it on and off from the seated position as well as being able to push the wheelchair manually.

The cost of an electric wheelchair is more than 3000 euros in most cases. The solution that is intended to be provided is the adaptation of an electric scooter to the wheelchair, transforming it into an electric one. Also, with a cost of 10 times lower than what exists in the market.

\section{Design}

For this design, the wheelchair's main wheels are taken off. The holes from the wheelchair wheel's axis are used to connect it to the developed design. This is the only modification which is done to the actual wheelchair. It is possible to assemble the wheelchair in its original form in a few minutes. The self-balancing scooter is used as an electrical motor and power source of this motorized wheelchair.

The self-balancing scooter, as seen in Figure 1, has a gyroscope system which can balance a person standing on it. It can be controlled through weight forth or back, which gets registered by pressure pads, located on the top of the standing platform. When rotating the pedal backwards the wheel will also revolve to the same direction. Pressure has to be applied on the pedals in order to control it. Both of the wheels are able to spin 
independently from each other, this allows a very tight turning radius. (CN204223088U, 2014)

In the design, almost the whole weight of the wheelchair and also the weight of the person sitting on it is located right over the scooter and directed to the pressure pads of the scooter. In that case, the controlling pedals are working correctly, the scooter's software measures that a person is standing on it.

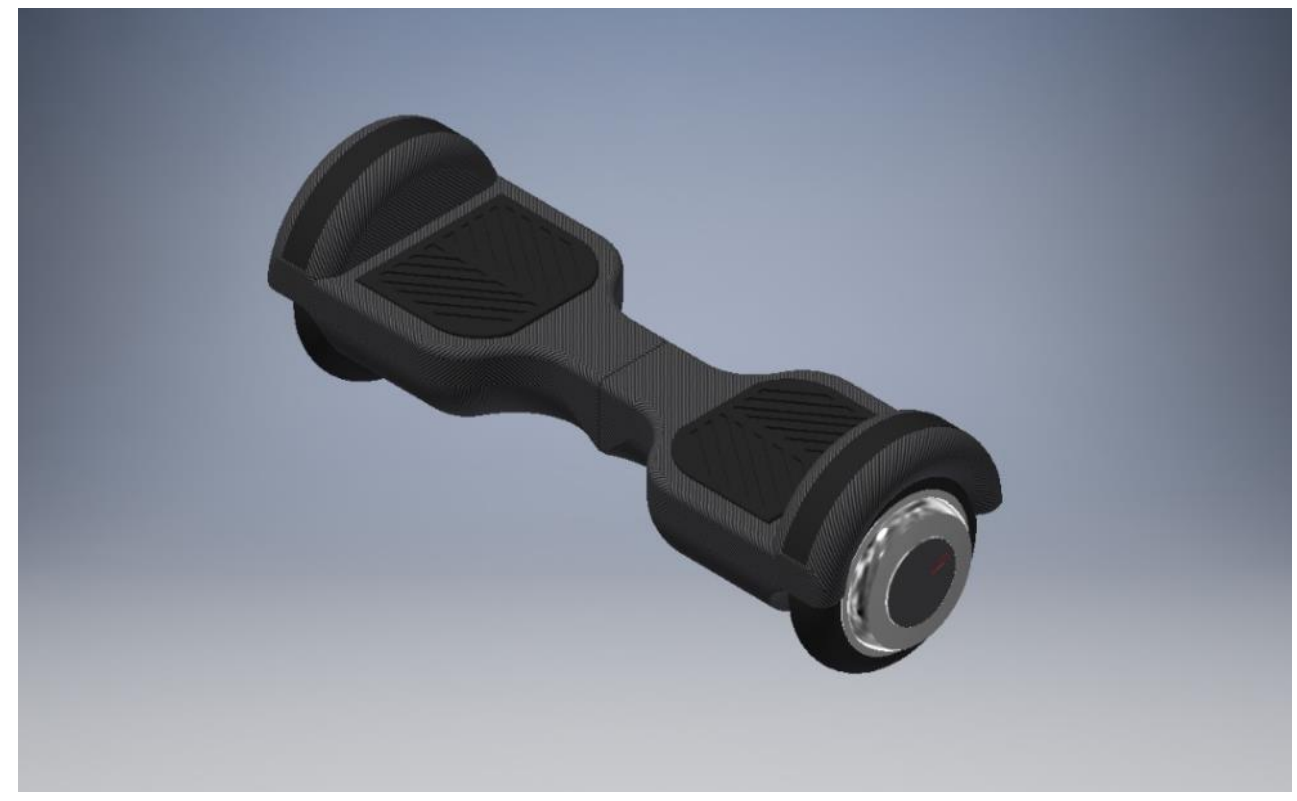

Fig. 1 A self-balancing scooter

The designed structure, which connects the wheelchair to the scooter, includes two mounting plates, one middle axis, a steering assist, and steering poles; the whole assembled design can be seen in figure 2 . The mounting plates are sitting on the pressure pads and an axis, which will be referred to as the middle axis, is connected to those mounting plates in a way that allows rotation. The steering assist refers to a spring-mechanism and has the purpose to return the control pedals back to a neutral position when the steering poles are untouched. The steering poles transmit movement to the steering assist, which will result in forwards motion when the pole is pushed forward and backwards motion in the opposite case. A detailed description of the parts will follow. This whole structure is connected to the wheelchair with bolts and nuts, the connection is fixed and it will not allow any rotation or sliding. 


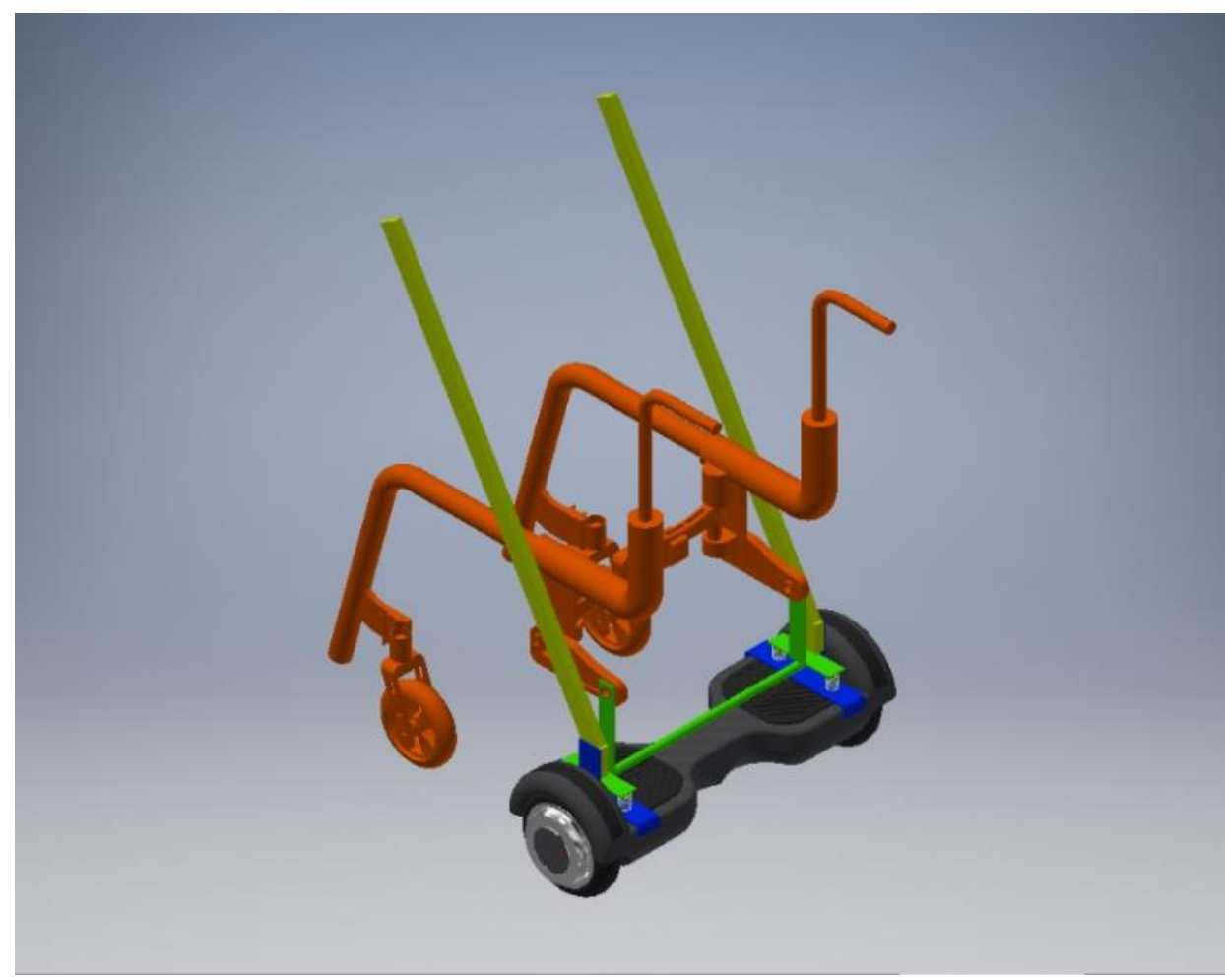

Fig. 2 Assembled structure

Using a self-balancing scooter which is able to turn around its own axis and placing it right under the wheelchair, allows the user to turn on the spot. Making it ideal for the use indoors and tight places like an elevator. Movement specifications like range and top speed depend on the used scooter and the wheelchair model. Manual pushing by another person is able when the scooter is switched off.

The self-balancing scooter itself is connected to the mounting plates with belts, which can be easily disconnected. The connection is pretty stable even without the belts because the shape of the mounting plate disables movement. Belts are added to the design to prevent detaching though lifting the wheelchair up or driving over obstacles. The mounting plate has foam on its lower side, so the scooter doesn't get scratched and avoids noises from vibrations. 
The main structure is made out of steel and the steering poles are made out of aluminum because they are not exposed to strong forces and it's lighter and therefore more comfortable for the user. Steel has been chosen for the rest of the construction because it's strong and can be easily modified. Almost all of the weight of the wheelchair and the user sits on the structure so it has to be made out of strong material. There are rubber grips on the steering poles to make them more comfortable to hold.

The Figure 3 shows displays the steering assist. One of the most critical parts is of this design is the steering assist. Without it the wheelchair would continue its movement indefinitely if the user loses the grip on the steering poles. If the user tries to move the steering poles he has to counter a force which is generated by springs at the steering assist. So when there is no external force affecting the poles, the system will return back to their neutral position.

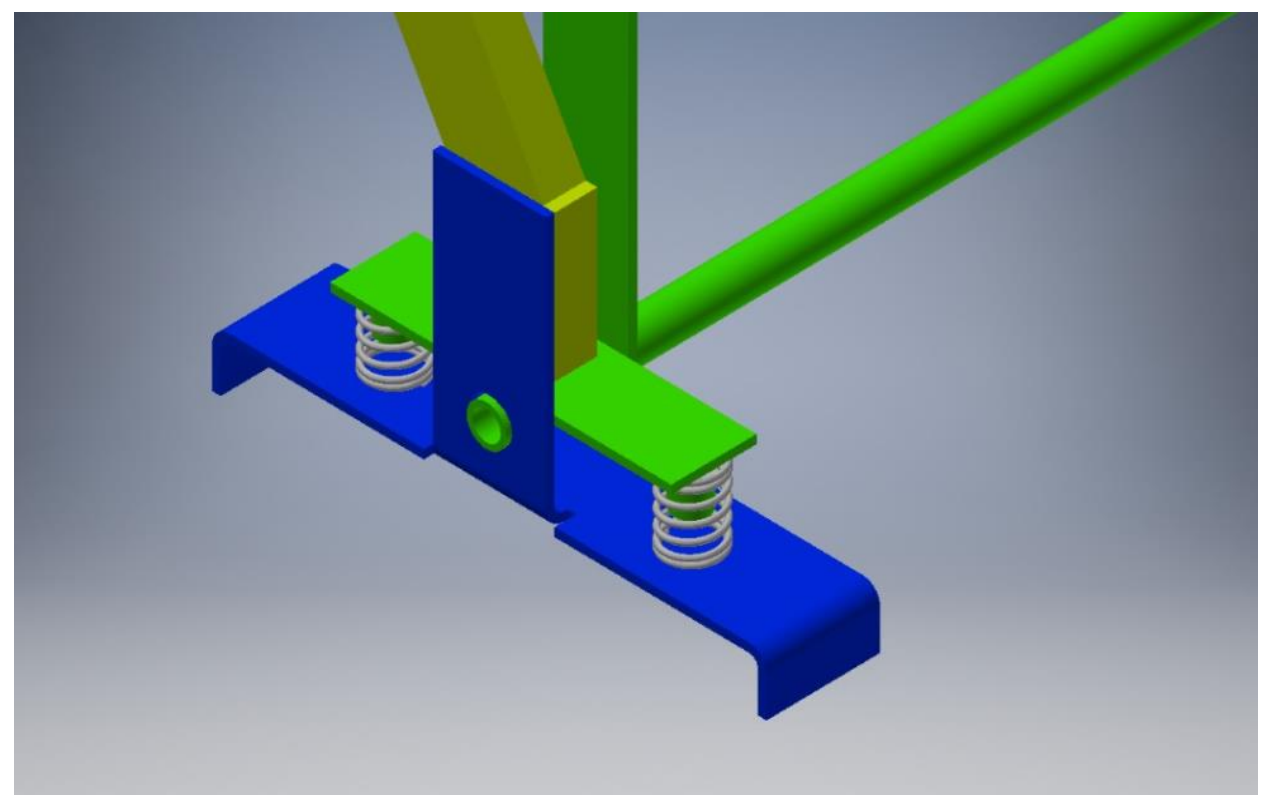

Fig. 3 Steering assist

Slowing down or stopping can be achieved by steering backwards. Meaning that the person in the wheelchair has to pull the steering sticks towards her-/himself. This will increase the pressure on the back of the pressure pads of the self-balancing scooter and it will slow down until the vertical position is reached. Further backwards steering will lead to moving backward. 
Various self-balancing scooters can be used as a power source, an advanced design of the mounting plates would allow them to be adjustable, so the user could change the width of the mounting plates. With this modification, the design does not require a specific scooter. Also with this improvement in mind, it's chosen to use adjustable belts as an attachment mechanism for the scooter.

\section{Conclusion}

With the presented design, a self-balancing scooter can be adapted to a conventional wheelchair. It is designed considering simplicity, efficiency and costs in order to be accessible and affordable for the maximum number of potential end-users.

The design has been tested and it is suitable for the indoor use as well as the outdoor use. The system (the wheelchair adapted with the electric scooter) is appropriate for people with arm mobility, but only requires a fraction of strength that would be necessary to push the wheelchair manually. This gives the opportunity to increase the autonomy of people, especially considering the increasing aging of the population in the future.

The system has been designed that it can be manufactured with relatively simple pieces such as springs, commercial scooter, welded iron plates, etc. Therefore, the option to create a business and to sell an all in one solution is given. Additional improvements could be an extra battery or an electronic interface for in reach of the end-user, where a charging plug and power switch could be located.

\section{Acknowledgements}

First and foremost, we would like to express our deep and sincere gratitude to our research supervisor Guillermo Peris-Fajarnés as well as Bernardo Pajares Moreno, María MonchoSantonja and Ismael Lengua for their support throughout our work. We would also like to give a huge thank you to Professor Pedro Fuentes by giving us his support and providing invaluable guidance throughout our project. Finally, a major thank you to the School of Engineering and Design, the Research Centre in Graphic Technologies and the Universidad Politècnica de Valencia for providing us with a workspace and allowing us to use their workshops. 
Benedikt Prusas, Karim Mansoor, L. Engelhardt, Saku Pirtilä, Bas Walgers, Louisa Lukoschek, Ismael Lengua, María Moncho-Santonja and Guillermo Peris-Fajarnés

\section{References}

Division., U. N. (2017). World Population Ageing. 2017. New York.

Foundation, W. (s.f.). www.wheelchairfoundation.org. Recuperado el 2019, de Analysis of wheelchair need.: https://www.wheelchairfoundation.org/fth/analysis-of-wheelchairneed/

Hollingshead, T. (2015). BYU students make the world's lightest, least expensive motorized wheelchair.

Hudakiva, A., \& Hornakova, A. (2011). Mobility and quality of life in elderly and geriatric patients. International Jounral of Nursing and Midwifery, 3(7). doi:ISSN 2441-2499

Organisation, W. H. (2015). World Report on Ageing and Health. doi:ISBN 978924 0694811

Patent. Two-wheel, self-balancing vehicle with independently movable foot placement sections (2014). Obtenido de https://patents.google.com/patent/CN204223088U/pt-PT

Winter, A. (2014). A cheap all-terrain wheelchair. Obtenido de https://www.thisismedtech.com/a-cheap-all-terrain-wheelchair/ 